\title{
Plasma Transport in
}

\section{Mixed Magnetic Topologies}

\author{
C. C. Hegna and J. D. Callen \\ Departments of Nuclear Engineering and Engineering Physics and Physics \\ University of Wisconsin
}

Madison, WI 53706-1687

\begin{abstract}
A simple model is introduced to illustrate some features concerning anomalous transport associated with magnetic turbulence. For magnetic topologies that are described as bands of stochasticity separated by regions with good flux surfaces, the transport coefficients deviate significantly from those describing completely stochastic magnetic fields. It is possible to have the electron heat diffusivity exceed a runaway electron diffusion coefficient, despite the existence of widespread magnetic stochasticity. Comparing the ratios of transport coefficients is not an accurate way to determine whether anomalous plasma transport is controlled by electrostatic or electromagnetic fluctuations.
\end{abstract}

PACS numbers: $\quad$ 52.25.Fi, 52.55.Dy, 52.35.Ra, 52.55.Pi 


\section{Introduction}

The mechanism of free streaming along stochastic magnetic field-lines is usually invoked to account for "radial" plasma transport from electromagnetic turbulence in toroidal magnetic confinement devices. Stochastic transport is characterized by a magnetic diffusion coefficient, $\mathrm{D}_{\mathbf{M}}$, that describes the magnetic field-line diffusion. The transport coefficients (e. g., the electron heat diffusivity, $\chi_{\mathrm{e}}$ ) are given by the product of $D_{M}$ and the appropriate particle velocity along the field lines. ${ }^{1}$ Since runaway electrons have the largest velocities, observations of energetic electron transport are used as a diagnostic for magnetic turbulence. ${ }^{2}$ If the magnetic stochasticity is uniform in the plasma volume of interest and causes the dominant transport process, the transport is characterized by

1) runaway electrons having the largest transport,

2) the ratio of the electron to the ion heat diffusivities equaling the ratio of the thermal velocities $\left(\chi_{\mathrm{i}} / \chi_{\mathrm{e}}=\mathrm{v}_{\mathrm{ti}} / \mathrm{v}_{\mathrm{te}}\right)$, and

3) the magnitude of the particle diffusion coefficient being about the same as the ion thermal diffusivity due to ambipolarity.

However, these conclusions would only hold if the magnetic topolegy describes uniform magnetic stochasticity. Alternatively, one might envision a magnetic topology that is described as a time-varying mix of "good" toroidal magnetic surfaces, stochastic regions and magnetic islands. ${ }^{3}$ For this more complicated magnetic topology, characterizing the plasma transport is a very difficult problem.

In this communication, we instead describe the plasma transport in a much simpler magnetic topology as a paradigmatic tool. We consider a steady-state topology that has two distinct regions of confinement, where spatially separated regions of magnetic stochasticity are imbedded in a plasma volume. (Possible magnetic island effects are discussed in Sec. III.) Correspondingly, where the magnetic stochasticity is present the associated magnetic diffusion of field-lines introduces a plasma transport 
mechanism in addition to those already present in the plasma. The localized magnetic stochasticity is not self-consistently derived. We consider two cases for the uniform transport. In the first case, we assume there is a collisional transport present throughout the plasma region. In the second case, we construct a simple model to describe anomalous transport caused by electrostatic turbulence present at all points in the plasma volume. With this simple model, predictions for the electron and ion heat diffusivities, particle and runaway diffusion coefficients are made for the hybrid magnetic topology.

\section{Magnetic Topology}

Consider a region of the plasma with "radial" extent L. In this region, suppose there exist transport processes so that temperature and density gradients exist. Additionally, there is a collection of slabs in this region that contain stochastic magnetic fields that produce additional transport processes localized inside the slabs, while the magnetic surfaces remain intact outside the slabs. The region of space with good magnetic surfaces, region $I$, has electron and ion heat diffusivities gi an by $\chi_{\mathrm{I}}^{e}$ and $\chi_{\mathrm{I}}^{\mathrm{i}}$, respectively. In region II, where magnetic stochasticity exists, the electron and ion heat diffusivities are given by $\chi_{\mathrm{II}}^{\mathrm{e}}$ and $\chi_{\mathrm{II}}^{\mathrm{i}}$, respectively. The fraction $\alpha$ is defined to be the radial extent in $\mathrm{L}$ that has good magnetic surfaces divided by $\mathrm{L}$, while $1-\alpha$ is the fraction of the plasma volume that is stochastic. The transport equations in I and II are given by

$$
\begin{aligned}
& \mathrm{q}_{\mathrm{e}}=-\chi_{\mathrm{III}}^{\mathrm{e}} \mathrm{n} \nabla \mathrm{T}_{\mathrm{e}}, \\
& \mathrm{q}_{\mathrm{i}}=-\chi_{\mathrm{III}}^{\mathrm{i}} \mathrm{n} \nabla \mathrm{T}_{\mathrm{i}}, \\
& \Gamma_{\mathrm{e}}=-\mathrm{D}_{\mathrm{III}}^{\mathrm{e}}\left[\nabla \mathrm{n}-\left(\mathrm{en} / \mathrm{T}_{\mathrm{e}}\right) \nabla \varphi\right], \\
& \Gamma_{\mathrm{e}}=-\mathrm{D}_{\text {III }}^{\mathrm{i}}\left[\nabla \mathrm{n}+\left(\mathrm{en} / \mathrm{T}_{\mathrm{i}}\right) \nabla \varphi\right],
\end{aligned}
$$

where $\nabla \varphi$ is the "radial" electric field which will be determined from ambipolarity and D denotes the particle diffusion coefficients. Assuming continuity of thermal flux and a 
linear temperature profile, total change in the electron temperature across regions I and II are given by $\Delta T_{e}^{I}=-\alpha L_{\mathrm{e}} \chi_{\mathrm{I}}^{\mathrm{e}} \mathrm{n}$ and $\Delta \mathrm{T}_{\mathrm{e}}^{\mathrm{II}}=-(1-\alpha) \mathrm{Lq}_{\mathrm{e}} \chi_{\mathrm{II}}^{\mathrm{e}} \mathrm{n}$, respectively. We can characterize the "global" transport across $\mathrm{L}$ by defining an effective electron thermal diffusivity by $\mathrm{q}_{\mathrm{e}}=-\chi_{\mathrm{eff}}^{\mathrm{e}} \mathrm{n} \Delta \mathrm{T}_{\mathrm{e}} / \mathrm{L}$. Using $\Delta \mathrm{T}_{\mathrm{e}}^{\mathrm{I}}+\Delta \mathrm{T}_{\mathrm{e}}^{\mathrm{II}}=\Delta \mathrm{T}_{\mathrm{e}}$, the effective thermal diffusivities are given by ${ }^{4}$

$$
\begin{aligned}
& \chi_{\text {eff }}^{e}=\frac{\chi_{I}^{e} \chi_{I I}^{e}}{\alpha \chi_{I I}^{e}+(1-\alpha) \chi_{I}^{e}}, \\
& \chi_{\text {eff }}^{i}=\frac{\chi_{I}^{i} \chi_{I I}^{i}}{\alpha \chi_{I I}^{i}+(1-\alpha) \chi_{I}^{i}},
\end{aligned}
$$

and similar expressions can be given for $D_{\text {eff }}^{e}$ and $D_{\text {eff }}^{i}$. Invoking the quasineutrality condition $\Gamma_{\mathrm{e}}=\Gamma_{\mathrm{i}}$, the effective particle diffusion coefficient can be derived and is given by the usual ambipolar formula

$$
D_{e f f}=\frac{D_{e f f}^{e} D_{e f f}^{i}\left(T_{e}+T_{i}\right)}{T_{i} D_{e f f}^{e}+T_{e} D_{e f f}^{i}}
$$

Equations (5)-(7) constitute the fundamental equations of this study.

As stated, in region II all the magnetic surfaces are destroyed so there is an additional transport process present due to the stochasticity. We write $\chi_{\mathrm{II}}^{\mathrm{e}}=\chi_{\mathrm{I}}^{\mathrm{e}}+v_{t e} \mathrm{D}_{M}$ and $\chi_{I I}^{i}=\chi_{I}^{i}+v_{t i} D_{M}$ where $D_{M}$ is the magnetic diffusion coefficient and $v_{t e}$ and $v_{t i}$ are the electron and ion thermal velocities. For the transport processes described by $\chi_{I}$ we examine two different possibilities. In the next section collisional processes are examined while in Sec. IIB transport due to electrostatic fluctuations is considered.

\section{A. Thermal plasma transport with collisional effects}

Transport theories based on collisional effects (e. g., neoclassical transport ${ }^{5}$ ) predict that the ion thermal diffusivity exceeds the electron thermal diffusivity by a factor 
of the square root of the mass ratios assuming $T_{e}=T_{i}$, and the particle diffusion coefficient is comparable to the electron thermal diffusivity.

In this section we reference all transport coefficients to the collisional value of the electron thermal diffusivity, $\chi_{\mathrm{C}}^{\mathrm{e}}$. We introduce the factor $\mathrm{A}$ in the stochastic region

$$
\mathrm{A} \equiv \frac{\mathrm{v}_{\mathrm{te}} \mathrm{D}_{\mathrm{M}}}{\chi_{\mathrm{C}}^{\mathrm{e}}},
$$

which describes the magnitude of the anomaly of the magnetic transport compared to the collisional value of the electron heat transport in region $\mathrm{I},: \chi_{\mathrm{II}}^{\mathrm{e}}=\chi_{\mathrm{I}}^{\mathrm{e}}(1+\mathrm{A})=\chi_{\mathrm{C}}^{\mathrm{e}}(1+\mathrm{A})$. Assuming equivalent temperatures, we write $\chi_{I}^{i}=\sqrt{m_{i} / m_{e}} \chi_{C}^{e}$ for the value of the collisional ion thermal diffusivity, and $\chi_{\mathrm{II}}^{\mathrm{i}}=\chi_{\mathrm{C}}^{\mathrm{e}}\left[\sqrt{\mathrm{m}_{\mathrm{i}} / \mathrm{m}_{\mathrm{e}}}+\mathrm{A} \sqrt{\mathrm{m}_{\mathrm{e}} / \mathrm{m}_{\mathrm{i}}}\right]$ in the stochastic region. Using Eqs. (5-7) we can express the transport quantities in terms of $A$ and $\alpha$, the magnitude and the extent of the anomaly produced by the magnetic stochasticity:

$$
\begin{aligned}
& \chi_{\text {eff }}^{e}=\chi_{C}^{e} \frac{A+1}{\alpha A+1}, \\
& \chi_{\text {eff }}^{i}=\chi_{C}^{e} \frac{A+m_{i} / m_{e}}{\alpha A \sqrt{m_{e} / m_{i}}+\sqrt{m_{i} / m_{e}}}=\chi_{C}^{i} \frac{A+m_{i} / m_{e}}{\alpha A+m_{i} / m_{e}} .
\end{aligned}
$$

In Figure 1, we plot the ratio of $\chi_{\text {eff }}^{i}$ to $\chi_{\text {eff }}^{e}$ versus $\alpha$ for the value $A=1000$ in a hydrogen plasma. Notice that as $\alpha \rightarrow 1$, the collisional value $\chi_{\mathrm{i}} / \chi_{\mathrm{e}}=\sqrt{\mathrm{m}_{\mathrm{i}} / \mathrm{m}_{\mathrm{e}}}$ is approached smoothly, and over a large range of $\alpha$ the ion diffusivity exceeds the electron diffusivity. For situations where there is a similar number of good magnetic surfaces as compared to destroyed surfaces, the transport is controlled by the properties of the good confinement region. At $\alpha=0$, the stochastic limit result is $\chi_{\mathrm{i}} / \chi_{\mathrm{e}}=\sqrt{\mathrm{m}_{\mathrm{e}} / \mathrm{m}_{\mathrm{i}}}\left[1+\mathrm{m}_{\mathrm{i}} / A \mathrm{~m}_{\mathrm{e}}\right]$ with $\mathrm{A} \gg 1$. At small $\alpha$, the ratio $\chi_{\mathrm{i}} \chi_{\mathrm{e}}$ is very sensitive to $\alpha$. The critical $\alpha$ at which $\chi_{\mathrm{i}}=\chi_{\mathrm{e}}$ is given by

$$
\alpha_{\text {crit }}=\frac{\sqrt{m_{i} / m_{e}} A-\sqrt{m_{i} / m_{e}}}{A}
$$


and is plotted in Fig. 2 versus $A$ for a hydrogen plasma. The critical $\alpha \rightarrow 0$ as $A \rightarrow \infty$ and obtains a maximum value as a function of $A$ at $\alpha_{c r i t} \cong \sqrt{m_{e} / m_{i}}$. Consequently, even when only a few good magnetic surfaces remain the ion transport can exceed the electron transport which is contrary to the extreme stochastic transport limit.

The effective particle diffusion coefficient is given by

$$
D_{\text {eff }}=2 \chi_{C}^{e} \frac{A^{2}+(A+1) m_{i} / m_{e}}{\alpha A^{2}+A\left(\sqrt{m_{i} / m_{e}}+\alpha m_{i} / m_{e}\right)+m_{i} / m_{e}} \text {. }
$$

In Fig. 3, $\chi_{\text {eff }}^{\mathrm{e}}, \chi_{\text {eff }}^{\mathrm{i}}$ and $\mathrm{D}_{\text {eff }}$ are plotted versus $\alpha$ for $A=1000$. The electron heat diffusivity is most sensitive to changes in $\alpha$ due to the fact that the electron transport changes most dramatically between region I and region II. The particle diffusion coefficient tracks the smaller of the heat diffusivities as $\alpha$ is varied due to the ambipolar property of the plasma transport.

\section{B. Thermal plasma transport with electrostatic effects}

In this section, we will assume that in addition to the localized magnetic transport stochasticity, there is a uniform bath of electrostatic turbulence that also causes anomalous transport. As a simple model we will assume that, since the transport is caused by radial $\tilde{\mathbf{E}} \times \mathbf{B}$ drifts which are independent of particle masses, region $I$ is described by $\chi_{\mathrm{I}}^{\mathrm{e}}=\chi_{\mathrm{I}}^{\mathrm{i}}=\mathrm{D}_{\mathrm{I}} \equiv \mathrm{D}_{\varphi}$. We define the anomaly factor

$$
A^{\prime} \equiv \frac{v_{t e} D_{M}}{D_{\varphi}}
$$

for the magnetic transport as compared to the electrostatic transport in region I. In region II, we now have $\chi_{\text {II }}^{e}=D_{\varphi}\left(1+A^{\prime}\right)$ and $\chi_{I I}^{i}=D_{\varphi}\left(1+A^{\prime} \sqrt{m_{e} / m_{i}}\right)$ for $T_{i}=T_{e}$. Clearly, for this case we will have $\chi_{\text {eff }}^{e} \geq D_{\text {eff }} \geq \chi_{\text {eff }}^{i}$ for all values of $\alpha$ since there is no region where ion transport exceeds electron transport. Using Eqs. (5-7) we get the effective diffusion coefficients 


$$
\begin{aligned}
& \chi_{\text {eff }}^{e}=D_{\varphi} \frac{A^{\prime}+1}{\alpha A^{\prime}+1}, \\
& \chi_{\text {eff }}^{i}=D_{\varphi} \frac{A^{\prime} \sqrt{m_{e} / m_{i}}+1}{\alpha A^{\prime} \sqrt{m_{e} / m_{i}}+1}, \\
& D_{e f f}=D_{\varphi} \frac{A^{\prime 2} \sqrt{m_{e} / m_{i}}+A^{\prime}+1}{\alpha A^{\prime} \sqrt{m_{e} / m_{i}}+A^{\prime}(1+\alpha) / 2+1},
\end{aligned}
$$

where we have used $\sqrt{\mathrm{m}_{\mathrm{e}} / \mathrm{m}_{\mathrm{i}}} \ll 1$. In Fig. 4 , these transport coefficients are plotted versus $\alpha$ with $A^{\prime}=1000$.

\section{Runaway electron transport}

Energetic electrons are the particles that are the most sensitive to magnetic fluctuations. These particles are also the least affected by collisional or electrostatic processes. Consequently, severe changes in the transport properties of the energetic electrons will be observed as the topology changes. As a simple model for the energetic electron transport in region $I$, we will assume that the diffusion coefficient is given by $D_{I}^{r}$ $=\chi_{\mathrm{I}}^{\mathrm{e}}\left(\mathrm{v}_{\mathrm{te}} / \mathrm{v}_{\mathrm{r}}\right)$ where $\mathrm{v}_{\mathrm{te}}$ and $\mathrm{v}_{\mathrm{r}}$ are the thermal and energetic electron velocities. For collisional processes this describes the fact that the collision frequency goes as the particle's energy to the negative three-halves power, while the step size goes as the particle velocity. For transport due to electrostatic turbulence, the diffusion coefficients are proportional to a characteristic correlation time. ${ }^{2}$ By estimating the time scale to be a transit time $(-\pi R / v)$, we get the above result. In region II, the diffusion coefficient is given by $D_{I I}^{r}=D_{I}^{r}+v_{r} D_{M}$ when transport due to the stochastic magnetic fields is included. Following the same procedure used to derive Eqs. (5) and (6), the effective energetic electron diffusion coefficient is derived and given by

$$
D_{\text {eff }}^{r}=\frac{D_{I}^{r} D_{I I}^{r}}{\alpha D_{I I}^{r}+(1-\alpha) D_{I}^{r}}
$$




$$
=\chi_{I}^{e} \sqrt{\frac{T_{e}}{E}} \frac{A+T_{e} / E}{\alpha A+T_{e} / E},
$$

where $E$ is the energetic electron energy and $A$ is the anomaly parameter defined in Eqs. (8) and (13) for the two different transport mechanisms of region I. In Fig. 5, the ratio $\mathrm{D}_{\mathrm{eff}}^{\mathrm{r}} / \chi_{\mathrm{eff}}^{\mathrm{e}}$ is plotted for a succession of $\alpha$ with $\mathrm{A}=100$ against $\mathrm{v}_{\mathrm{r}} / \mathrm{v}_{\mathrm{te}}$. For the completely stochastic limit $\alpha=0$, given by Fig. $5 \mathrm{~d}, \mathrm{D}_{\mathrm{eff}}^{\mathrm{r}} / \chi_{\mathrm{eff}}^{\mathrm{e}}$ increases monotonically with $\mathrm{v}_{\mathrm{r}} / \mathrm{v}_{\mathrm{te}}$ as expected from stochastic transport. However, as shown in Figs. 5a - 5c, even for a very small number of remaining magnetic surfaces, the energetic electron diffusion drops dramatically in comparison to the electron heat diffusivity. The value of $\alpha$ at which

$D_{\text {eff }}^{r} \chi_{\text {eff }}^{e}=1$ is given approximately by $\alpha_{r}=A^{-1} \sqrt{T_{e} / E}$ for $A \gg 1$ and $E>T_{e}$. From Eq. (11), it can be seen that $\alpha_{\mathrm{r}}<\alpha_{\text {crit }}$ for $\sqrt{m_{i} / m_{e}}>\sqrt{T_{e} / E}\left(1+m_{i} / A m_{e}\right) /\left(1-A^{-1} \sqrt{m_{i} / m_{e}}\right)^{-1}$. It is therefore easy to construct situations where $\chi_{\text {eff }}^{e}>\chi_{\text {eff }}^{i}$ and $\chi_{\text {eff }}^{e}>D_{\text {eff }}^{r}$. In this case, the magnetic stochasticity is widespread enough that the electron heat diffusivity exceeds the ion heat diffusivity but that the runaway diffusion is smaller than the thermal plasma heat transport due to a small number of good surfaces where the energetic electron transport is very small.

\section{Discussion}

Equations (5-7) and (17) describe the diffusion coefficients of a plasma that has a magnetic topology which contains both regions with robust magnetic surfaces and localized regions of magnetic stochasticity. Thermal electron heat transport is much more sensitive than ion transport to slight variations in the strength and extent of the magnetic stochasticity, particularly in the limit $A \gg 1$ (very anomalous transport along stnchastic field lines) and $\alpha<1$ (very few good magnetic surfaces). However, even for small values of $\alpha$ (mostly stochastic magnetic fields), the ion heat diffusivity can exceed the electron heat diffusivity when $\alpha>\alpha_{\text {crit }}$ [see, Eq. (11) and Fig. 2] in plasmas where collisional processes control the transport in the non-stochastic field region. For the 
collisional case, the anomalous ion heat diffusivity varies only from $\chi_{\text {eff }}^{i}=\chi_{C}^{i}$ to $\chi_{e f f}^{i}=$ $\chi_{\mathrm{C}}^{\mathrm{i}}\left(1+A \mathrm{~m}_{\mathrm{e}} / \mathrm{m}_{\mathrm{i}}\right)$ so that significant enhancement can only be modeled if the anomaly amplitude $A$ is large compared to the ratio of the ion to electron mass. In particular, if we take the limit of infinite transport in the stochastic region $(A \rightarrow \infty)$, the transport coefficients are given by $\chi_{\text {eff }}^{e}=\chi_{C}^{e} / \alpha$ and $\chi_{\text {eff }}^{i}=\chi_{C}^{i} / \alpha$; for this case the transport is controlled by what happens on the good magnetic surfaces. In the case that electrostatic transport describes the transport in the non-stochastic region, the transport coefficients obey $\chi_{\text {eff }}^{\mathrm{e}} \geq \mathrm{D}_{\text {eff }} \geq \chi_{\text {eff }}^{\mathrm{i}}$ for the model used here. It is worth noting that our simple model for the electrostatic fluctuations (described in the first paragraph of Sec. IIB) is not generic to all types of electrostatic transport. Runaway electron confinement shows a large sensitivity to the extent of the magnetic stochasticity because energetic electrons have such small transport in regions where the magnetic surfaces are robust. Thus it is possible to have the electron heat diffusivity exceed the runaway diffusion coefficient when widespread (but not complete) magnetic stochasticity is present, in sharp contrast to the purely stochastic transport limit.

The present model is quite primitive and does not include a large number of effects. Implicitly, it is assumed that the widths of the stochastic slabs and the widths of the good surface regions exceed any characteristic particle orbit width (e. g., gyroradius or banana width) so that we may use a fluid treatment to describe the transport. Additionally, the effect of having coherent magnetic islands imbedded in this topology has been ignored. The effect of a single magnetic island on the local density and temperature profiles has recently been examined, where it is found that a flattering of the profiles inside the island separatrix is a very good approximation. ${ }^{6}$ A model based upon this effect of an island has indicated that the energy confinement time is reduced by a factor proportional to the plasma cross-sectional area occupied by the island, ${ }^{7}$ which is supported by some experimental evidence. ${ }^{8}$ A possible modification of the present model to include island effects would then be to consider transport through three different 
types of regions: stochastic regions, regions of good magneti s surfaces, and regions with magnetic islands where the regions within the magnetic islands provide virtually no insulation against radial transport. Such a model would yield transport coefficients larger than those indicated here by the inverse of the fraction of the plasma volume that does not contain contain islands. More generally, if the plasma volume was described by a set of transport processed that produce diffusion coefficients $\chi_{\mathrm{k}}$ and each process is localized to a fractional region $f_{k}$ of the plasma $\sum_{k} f_{k}=1$ ], then the effective transport coefficient would be given by $\chi_{\text {eff }}=\left[\sum_{k}\left(f_{k} / \chi_{k}\right)\right]^{-1}$.

The present analysis has considered a magnetic topology that is steady-state. Time dependent processes, such as growing islands and the associated magnetic stochasticity, ${ }^{3}$ can produce additional transport that is not accounted for here. Also, as indicated by the analysis presented in this letter, it is very difficult to anticipate the ratios of the various transport coefficients for a complex mix of good, stochastic and magnetic island regions in a steady-state situation, let alone when they are time-varying.

Despite the simplicity of the present model, it is clear from it and the preceding discussion that if the confining magnetic topology of a plasma consists of a mix of good, stochastic and magnetic island regions, the ratio of any two transport coefficients is not sufficient to distinguish between electrostatic and electromagnetic models of anomalous plasma transport. In particular, the ratio of the runaway electron to electron heat diffusisvity can be less than unity even with large regions of stochasticity and concomitant anomalous electron heat transport. Rather, it would seem that except for extreme limiting cases, the ratio of various transport coefficients is not generic to electromagnetic fluctuation induced transport, but instead must be determined from the fundamental nature of the underlying fluctuations. 


\section{Acknowledgments}

C. C. H. would like to acknowledge discussions with the participants of the Cargèse 1992 Workshop on Magnetic Turbulence and Transport. This work is supported by the U. S. Department of Energy under grant no. DE-FG02-86ER53218. 


\section{References}

1. A. B. Rechester and M. N. Rosenbluth, Phys. Rev. Lett. 40, 78 (1978).

2. J. R. Myra, P. J. Catto, A. J. Wootton, R. D. Bengston, and P. W. Wang, Phys. Fluids B 4, 2092 (1992) and references within.

3. C. C. Hegna and J. D. Callen, Phys. Fluids B 4, 1855 (1992); C. C. Hegna and J.

D. Callen, to appear in Proceedings of the International Conference on New Ideas in Tokamak Confinement (La Jolla, 1992), Ed. V. Stefan.

4. P. Beaufume, M. A. Dubois, and M. S. Mohamed Benkadda, Phys. Lett. A 147, 87 (1990).

5. F. L. Hinton and R. L. Hazeltine, Rev. Mod. Phys. 48, 239 (1976): S. P. Hirshman and D. J. Sigmar, Nucl. Fusion 21, 1079 (1981).

6. C. C. Hegna and J. D. Callen, Phys. Fluids B 4, 4072, (1992).

7. Z. Chang and J. D. Callen, Nucl. Fusion 30, 219 (1990).

8. J. A. Snipes, D. J. Campbell, T. C vender, M. von Hellerman, and H. Weisen, Nucl. Fusion 30, 205 (1990). 


\section{Figure Captions}

Fig. 1 - The ratio $\chi_{\mathrm{i}} / \chi_{\mathrm{e}}$ versus $\alpha$ for the anomaly factor $\mathrm{A}=1000$ where collisional processes determine the transport in re,$\Omega I$.

Fig. 2 - The critical $\alpha$ versus $A$ for which $\chi_{i}=\chi_{e}$.

Fig. 3 - The transport coefficients $\chi_{e}, \chi_{i}$, and D versus $\alpha$ with $A=1000$ when collisional processes determine the transport in region $I$.

Fig. 4 - The transport coefficients $\chi_{e}, \chi_{i}$, and D versus $\alpha$ with $A^{\prime}=1000$ when electrostatic fluctuations determine the transport in region $I$.

Fig. 5 - The ratio $D_{\mathrm{ff}}^{\mathrm{r}} \chi_{\text {eff }}^{e}$ versus the ratio of particle velocities $v_{\mathrm{r}} / v_{\text {te }}$ with $A=100$ for the four cases: a) $\alpha=0.02$, b) $\alpha=0.002$, c) $\alpha=0.0002$ and d) $\alpha=0$ (the pure stochasticity limit). 


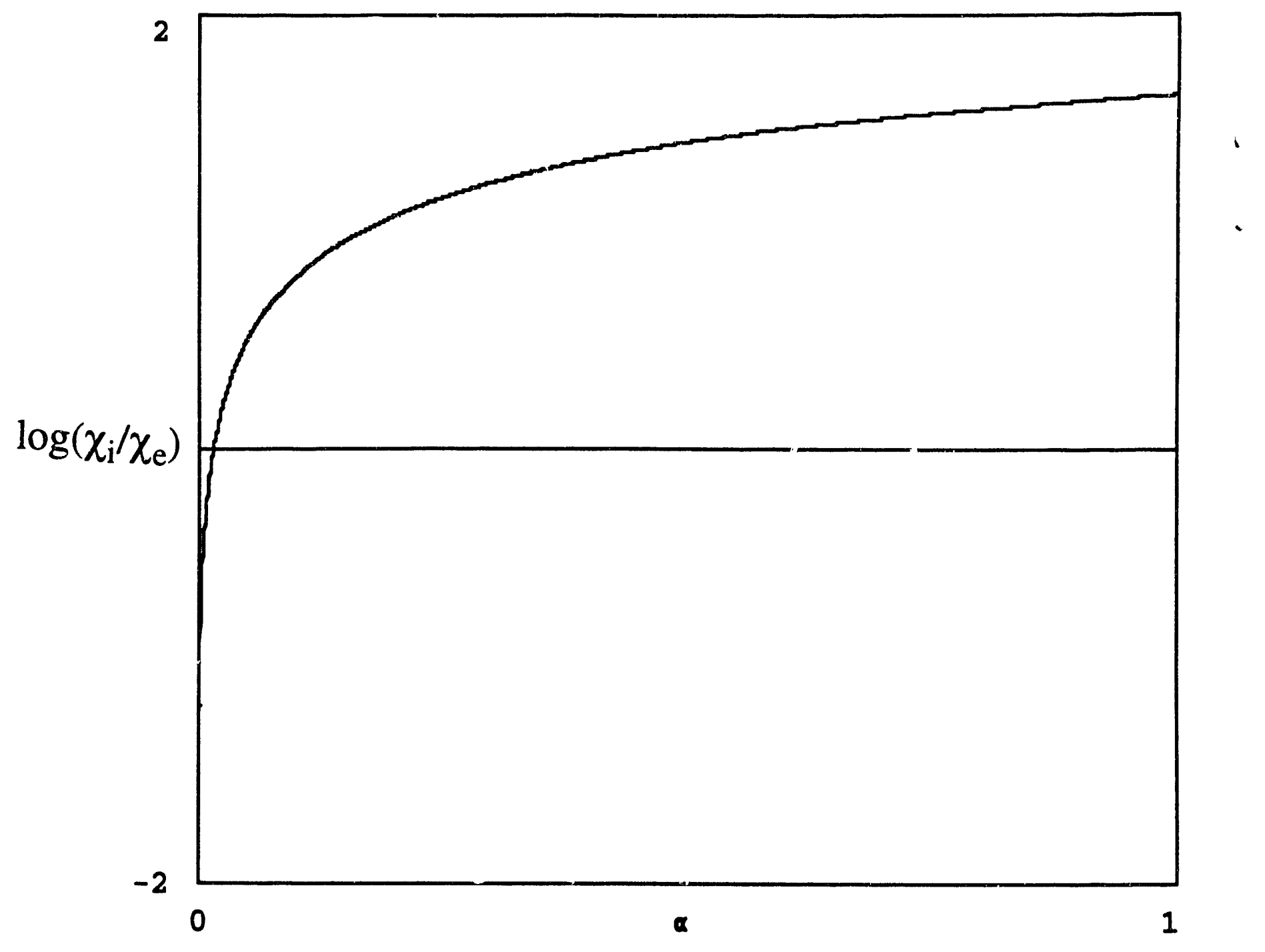

Fig. 1 


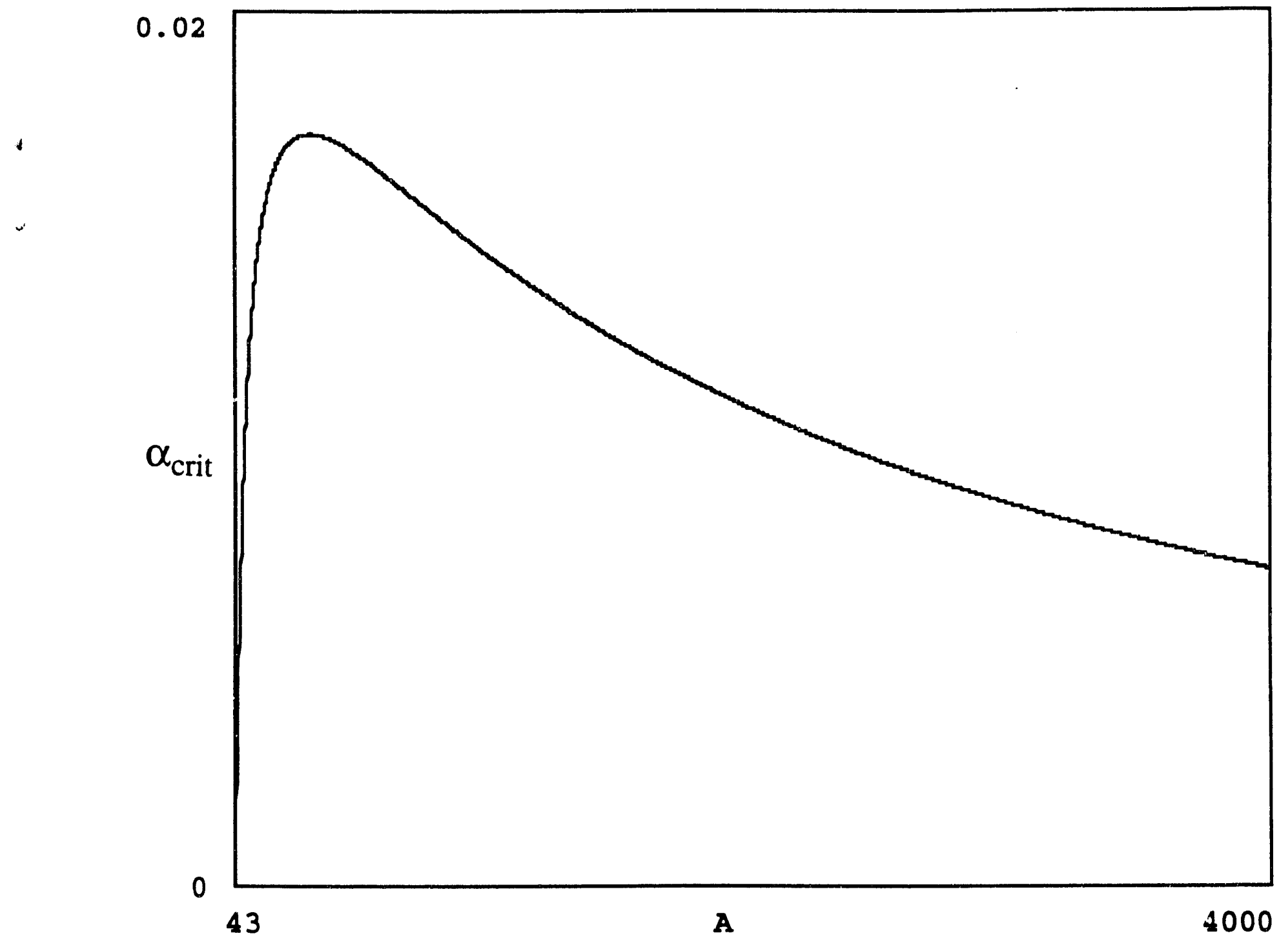

Fig. 2 


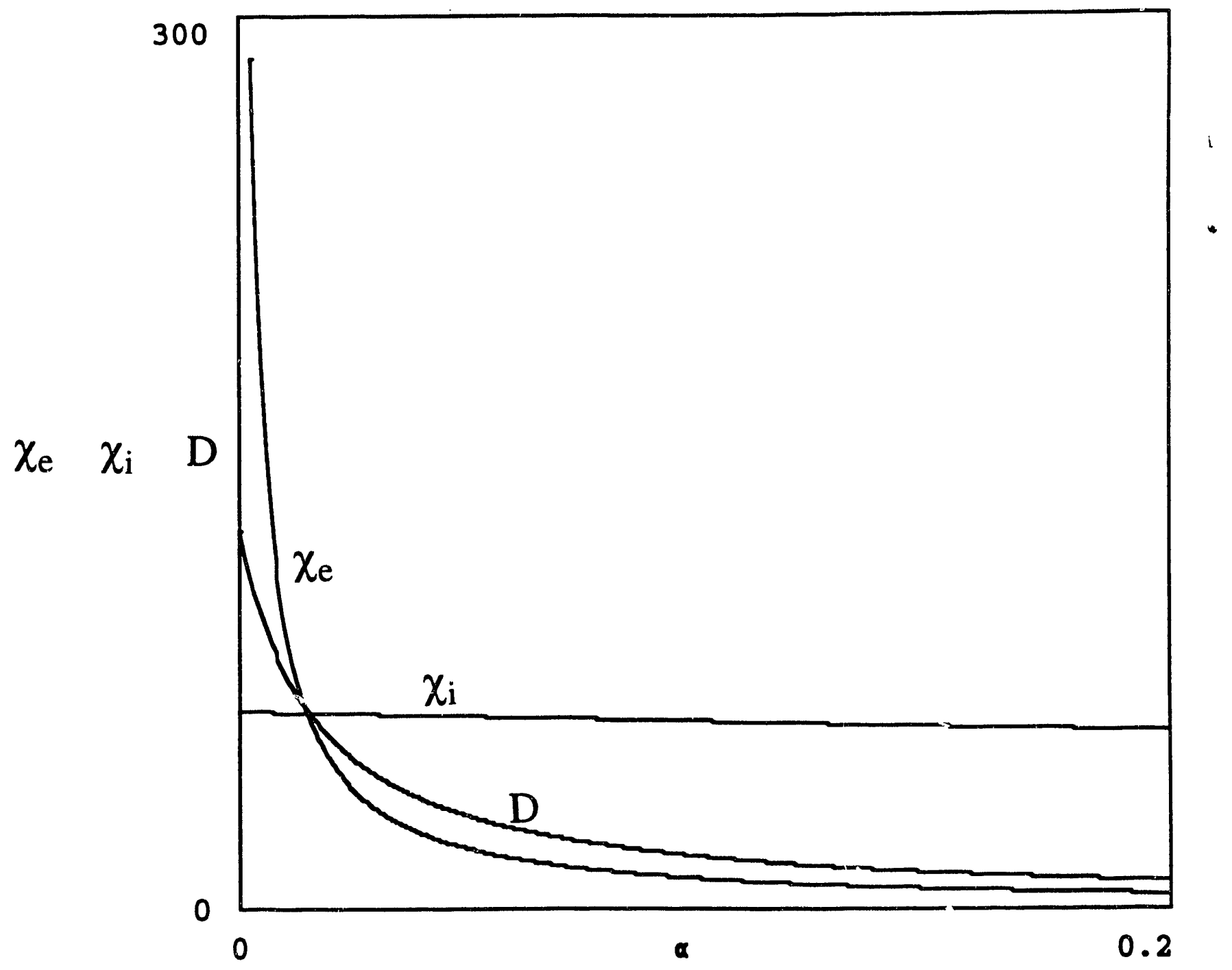

Fig. 3 




Fig. 4 


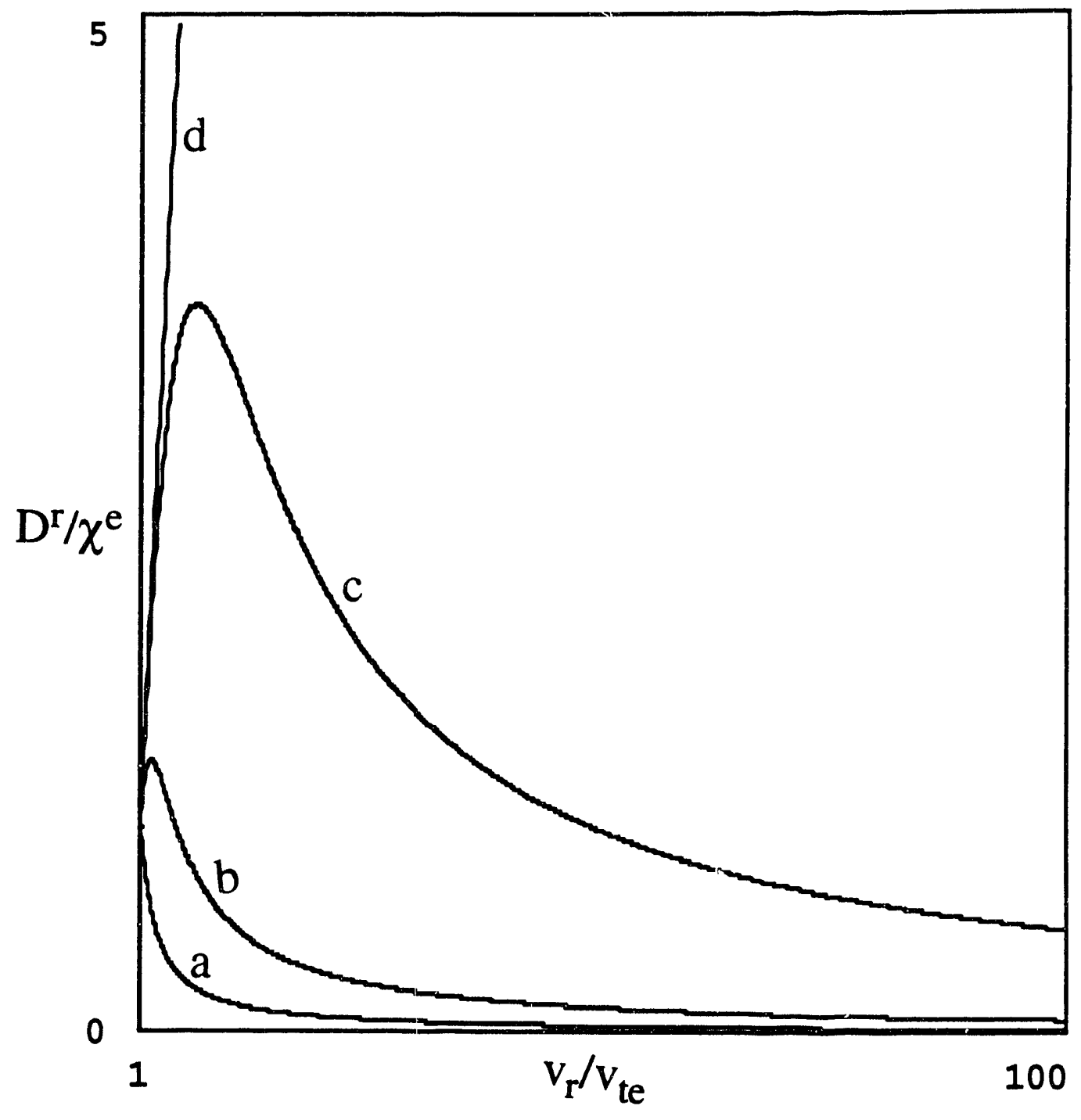

Fig. 5 


